\title{
Resolutions of Identity for Some Non-Hermitian Hamiltonians. I. Exceptional Point in Continuous Spectrum ${ }^{\star}$
}

\author{
Alexander A. ANDRIANOV ${ }^{\dagger \ddagger}$ and Andrey V. SOKOLOV ${ }^{\dagger}$ \\ $\dagger$ V.A. Fock Department of Theoretical Physics, Sankt-Petersburg State University, \\ 198504 St. Petersburg, Russia \\ E-mail: andrianov@bo.infn.it, avs_avs@rambler.ru \\ $\ddagger$ ICCUB, Universitat de Barcelona, 08028 Barcelona, Spain \\ E-mail: andrianov@icc.ub.edu
}

Received August 06, 2011, in final form November 25, 2011; Published online December 05, 2011 http://dx.doi.org/10.3842/SIGMA.2011.111

\begin{abstract}
Resolutions of identity for certain non-Hermitian Hamiltonians constructed from biorthogonal sets of their eigen- and associated functions are given for the spectral problem defined on entire axis. Non-Hermitian Hamiltonians under consideration possess the continuous spectrum and the following peculiarities are investigated: (1) the case when there is an exceptional point of arbitrary multiplicity situated on a boundary of continuous spectrum; (2) the case when there is an exceptional point situated inside of continuous spectrum. The reductions of the derived resolutions of identity under narrowing of the classes of employed test functions are revealed. It is shown that in the case (1) some of associated functions included into the resolution of identity are normalizable and some of them may be not and in the case (2) the bounded associated function corresponding to the exceptional point does not belong to the physical state space. Spectral properties of a SUSY partner Hamiltonian for the Hamiltonian with an exceptional point are examined.
\end{abstract}

Key words: non-Hermitian quantum mechanics; supersymmetry; exceptional points; resolution of identity

2010 Mathematics Subject Classification: 81Q60; 81R15; 47B15

\section{Introduction}

The interest to exceptional points in non-Hermitian quantum dynamical systems has been revoked recently $[1,2,3,4,5]$ although their very notion exists quite a time $[6,7,8,9,10]$. Their appearance can be associated to level coalescence at complex coupling constants for initially Hermitian Hamiltonians [11]. If existing they play an important role in definition of energy spectra and in construction of biorthogonal bases in Riesz spaces [12].

Whereas the appearance of exceptional points in discrete spectrum does not give rise to any principal obstacles for building a resolution of identity, the emergence of exceptional points inside or on the border of continuous spectrum makes the very construction of resolutions of identity rather sophisticated [2]. Their correct description in brief represents the main aim of our paper (entitled as Part I) whereas in the subsequent paper [13] (entitled as Part II) the detailed proofs of the results announced here are presented by one of us (A.V.S.).

Let us start with the notion of exceptional point and further on outline the structure of the present work. The spectrum of a Hermitian Hamiltonian, in general, consists of continuous part

${ }^{\star}$ This paper is a contribution to the Proceedings of the Workshop "Supersymmetric Quantum Mechanics and Spectral Design" (July 18-30, 2010, Benasque, Spain). The full collection is available at http://www.emis.de/journals/SIGMA/SUSYQM2010.html 
and discrete points. Meanwhile the spectrum of a non-Hermitian Hamiltonian may contain also a new type of spectral points embedded into a continuous spectrum and/or into a discrete one, namely, exceptional points.

The exceptional point of the spectrum of one-dimensional Hamiltonian $h$ defined on entire axis is an eigenvalue $\lambda_{0}$ of this Hamiltonian for which there is a normalizable eigenfunction $\psi_{0}(x)$ and also a number of associated functions [7] $\psi_{j}(x), j=1, \ldots, n-1$ :

$$
h \psi_{0}=\lambda_{0} \psi_{0}, \quad\left(h-\lambda_{0}\right) \psi_{j}=\psi_{j-1}, \quad j=1, \ldots, n-1 .
$$

For a discrete spectrum the latter ones are typically normalizable [2, 3]. On the other hand some non-normalizable associated functions bounded or even growing at infinity may be involved in building of resolution of identity as well. It will be proven in Part II. The number $n$ is an algebraic multiplicity of $\lambda_{0}$. Thus, in continuous spectrum one can deal with two types of algebraic multiplicities (which are not necessarily equal: their different types for continuous spectrum are discussed in conclusions). If an exceptional point $\lambda_{0}$ belongs to a discrete part of the spectrum then $n$ simultaneously characterizes the order of a pole at $E=\lambda_{0}$ of the Green function. For an exceptional point $\lambda_{0}$ on the border of continuous spectrum the Green function reveals (see Section 2.3) a branching point with the pole order $2 n+1$ in the variable $\sqrt{E-\lambda_{0}}$, where $n$ is a maximal number of linearly independent eigen- and formal associated functions of $h$ for an eigenvalue $\lambda_{0}$ in the resolution of identity. When an exceptional point lies inside of continuous part of the spectrum the pole order may be larger than $n$ (which has the same meaning as in the previous sentence) that is elucidated in details in conclusions.

In this paper we build resolutions of identity for certain non-Hermitian Hamiltonians constructed from biorthogonal sets of their eigen- and associated functions for the spectral problem defined on entire axis. Non-Hermitian Hamiltonians under consideration are taken with continuous spectrum and the following peculiarities are investigated: in Section 2 the case when there is an exceptional point of arbitrary multiplicity situated on a boundary of continuous spectrum; in Section 3 the case when there is an exceptional point inside of continuous spectrum. In Section 4 in conclusions the different ways to introduce algebraic multiplicities are discussed and the SUSY tools $[14,15,16,17,18,19,20,21,22]$ in regulating them are inspected.

More specifically in Sections 2 and 3 the reductions of the resolutions of identity under narrowing of the classes of employed test functions are elaborated. It is shown that the bounded associated function in an exceptional point inside of continuous spectrum does not belong to the physical state space (i.e. does not belong to the complete biorthogonal system built from eigenfunctions of the Hamiltonian and cannot be reproduced with the help of harmonic expansion generated by an appropriate resolution of identity). If an exceptional point lies on a boundary

of continuous spectrum then some of associated functions included into the resolution of identity are normalizable and some of them may be not, still being elements of a rigged Hilbert space [23] and its dual one, the Gelfand triple generalized onto biorthogonal resolutions of identity.

\section{Resolutions of identity for model Hamiltonians with an exceptional point of arbitrary multiplicity at the bottom of continuous spectrum}

\subsection{Basic constructions}

Let us consider the sequence of Hamiltonians,

$$
h_{n}=-\partial^{2}+\frac{n(n+1)}{(x-z)^{2}}, \quad x \in \mathbb{R}, \quad \partial \equiv \frac{d}{d x}, \quad \operatorname{Im} z \neq 0, \quad n=0,1,2, \ldots,
$$


where $h_{0}$ is the Hamiltonian of a free particle and all these Hamiltonians are $\mathcal{P} T$-symmetric [24] for the choice $\operatorname{Re} z=0$. One can easily check that for the Hamiltonian $h_{n}$ on the energy level $E=0$ there is an eigenfunction $\psi_{n 0}(x)$ and a chain of formal associated functions $\psi_{n l}(x)$ :

$$
\begin{aligned}
& h_{n} \psi_{n 0}=0, \quad h_{n} \psi_{n l}=\psi_{n, l-1}, \quad l=1,2,3, \ldots, \\
& \psi_{n 0}(x)=\frac{(-i)^{n}(2 n-1) ! !}{\sqrt{2 \pi}(x-z)^{n}}, \quad \psi_{n l}(x)=\frac{(-i)^{n}(2 n-2 l-1) ! !}{\sqrt{2 \pi}(2 l) ! !(x-z)^{n-2 l}}, \quad l=0,1,2, \ldots, \\
& 0 ! !=(-1) ! !=1, \quad(-2 m-1) ! !=\frac{(-1)^{m}}{(2 m-1) ! !}, \quad m=1,2,3, \ldots
\end{aligned}
$$

Moreover for odd $n$ the functions $\psi_{n l}(x) ; l=0, \ldots,[n / 2]$ are normalizable (i.e. belong to $L^{2}(\mathbb{R})$ ) and when $l>[n / 2]$ they are non-normalizable and unboundedly growing for $x \rightarrow \pm \infty$. For even $n$ the functions $\psi_{n l}(x) ; l=0, \ldots,[n / 2]-1$ are normalizable, the function

$$
\psi_{n, n / 2}(x) \equiv \frac{(-i)^{n}(n-1) ! !}{\sqrt{2 \pi} n ! !}
$$

is bounded but non-normalizable and the functions $\psi_{n l}(x)$ for $l>n / 2$ are non-normalizable and unboundedly growing for $x \rightarrow \pm \infty$.

The Hamiltonians $h_{n}, n=0,1,2, \ldots$ are intertwined by the operators

$$
q_{n}^{ \pm}=\mp \partial-\chi_{n}(x), \quad \chi_{n}(x)=\frac{\psi_{n 0}^{\prime}(x)}{\psi_{n 0}(x)} \equiv-\frac{n}{x-z}, \quad q_{n}^{-} \psi_{n 0}=0, \quad n=0,1,2, \ldots,
$$

with the help of the chain (ladder) construction $[25,26,27,17]$

$$
h_{n} q_{n}^{+}=q_{n}^{+} h_{n-1}, \quad q_{n}^{-} h_{n}=h_{n-1} q_{n}^{-}
$$

and

$$
h_{n}=q_{n}^{+} q_{n}^{-}, \quad h_{n-1}=q_{n}^{-} q_{n}^{+}, \quad n=0,1,2, \ldots, \quad h_{-1}=-\partial^{2}=h_{0} .
$$

One easily check that

$$
q_{n}^{-} \psi_{n l}=-i \psi_{n-1, l-1}, \quad n, l=1,2,3, \ldots
$$

As well the eigenfunctions $\psi_{n}(x ; k)$ for continuous spectrum of the Hamiltonian $h_{n}$ can be produced from the eigenfunctions

$$
\psi_{0}(x ; k)=\frac{1}{\sqrt{2 \pi}} e^{i k x}, \quad k \in \mathbb{R}
$$

for the continuous spectrum of the Hamiltonian $h_{0}$ of a free particle with the help of intertwining operators $(2.3)$ :

$$
\begin{aligned}
& \psi_{n}(x ; k)=\frac{1}{\sqrt{2 \pi}}\left(\frac{i}{k}\right)^{n} q_{n}^{+} \cdots q_{1}^{+} e^{i k x} \\
& h_{n} \psi_{n}(x ; k)=k^{2} \psi_{n}(x ; k), \quad k \in(-\infty, 0) \cap(0,+\infty), \quad n=0,1,2, \ldots
\end{aligned}
$$

For the function $\psi_{n}(x ; k)$ one can derive the following explicit representation by induction,

$$
\psi_{n}(x ; k)=\frac{1}{\sqrt{2 \pi}} e^{i k x} \sum_{m=0}^{n} \frac{(n+m) !}{2^{m} m !(n-m) !} \frac{i^{m}}{k^{m}(x-z)^{m}},
$$


where from it follows that the cofactor $(i / k)^{n}$ in $(2.5)$ provides the asymptotic form for

$$
\psi_{n}(x ; k)=\frac{1}{\sqrt{2 \pi}} e^{i k x}\left[1+O\left(\frac{1}{x}\right)\right], \quad x \rightarrow \pm \infty .
$$

As well, using (2.3) and (2.5), we can get the following representations for $\psi_{n}(x ; k)$ :

$$
\begin{aligned}
\psi_{n}(x ; k) & =\left.\frac{1}{\sqrt{2 \pi}} e^{i k z}\left(\frac{d}{d t}-\frac{n}{t}\right)\left(\frac{d}{d t}-\frac{n-1}{t}\right) \cdots\left(\frac{d}{d t}-\frac{1}{t}\right) e^{t}\right|_{t=i k(x-z)} \\
& =\frac{e^{i k z}}{\sqrt{2 \pi} i^{n}(x-z)^{n}}\left(\frac{\partial}{\partial k}-\frac{n}{k}\right)\left(\frac{\partial}{\partial k}-\frac{n-1}{k}\right) \cdots\left(\frac{\partial}{\partial k}-\frac{1}{k}\right) e^{i k(x-z)} .
\end{aligned}
$$

At last, it follows from (2.5) and (2.8) that

$$
\begin{aligned}
\psi_{n}(x ; k) & =\frac{i}{k} q_{n}^{+} \psi_{n-1}(x ; k) \\
& =\frac{e^{i k z}}{i(x-z)}\left(\frac{\partial}{\partial k}-\frac{n}{k}\right)\left[e^{-i k z} \psi_{n-1}(x ; z)\right], \quad n=1,2,3, \ldots
\end{aligned}
$$

Let us find now the connection between $\psi_{n}(x ; k)$ and $\psi_{n l}(x), l=0,1,2, \ldots$ For this purpose we calculate the following derivative, using Leibnitz formula and the relation 4.2.7.14 from [28],

$$
\begin{aligned}
\lim _{k \rightarrow 0} & \frac{\partial^{m}}{\partial k^{m}}\left[e^{-i k z} k^{n} \psi_{n}(x ; k)\right]=\left.\frac{\partial^{m}}{\partial k^{m}}\left[\frac{1}{\sqrt{2 \pi}} e^{i k(x-z)} \sum_{s=0}^{n} \frac{(n+s) !}{2^{s} s !(n-s) !} \frac{i^{s} k^{n-s}}{(x-z)^{s}}\right]\right|_{k=0} \\
= & \frac{i^{n+m} n !}{\sqrt{2 \pi} 2^{n}(x-z)^{n-m}} \sum_{j=0}^{\min \{m, n\}}(-2)^{j} C_{m}^{j} C_{2 n-j}^{n}=\frac{i^{n+m}}{\sqrt{2 \pi}(x-z)^{n-m}} \prod_{s=1}^{n}(2 s-m-1) \\
& = \begin{cases}(-1)^{n} m ! \psi_{n, m / 2}(x), & m \text { is even, } \\
0, & m \text { is odd and } \leqslant 2 n-1, \\
\frac{i^{m-n}(m-1) ! !}{\sqrt{2 \pi}(m-2 n-1) ! !} \frac{1}{(x-z)^{n-m}}, & m \text { is odd and }>2 n-1,\end{cases}
\end{aligned}
$$

where $C_{n}^{m} \equiv n ! /[m !(n-m) !]$ is a binomial coefficient. Thus,

$$
\psi_{n l}(x)=\frac{(-1)^{n}}{(2 l) !} \lim _{k \rightarrow 0} \frac{\partial^{2 l}}{\partial k^{2 l}}\left[e^{-i k z} k^{n} \psi_{n}(x ; k)\right], \quad l=0,1,2, \ldots
$$

Since the continuous spectrum of the Hamiltonian $h_{n}, n=1,2,3, \ldots$ coincide with $[0,+\infty)$ (see (2.5)), the eigenvalue $E=0$ of this Hamiltonian for the chain of functions $\psi_{n l}(x), l=$ $0,1,2, \ldots$ is situated at the bottom of continuous spectrum. It will be shown in Section 2.3 which of these functions are included in the resolution of identity constructed from eigen- and associated functions of $h_{n}$.

\subsection{Biorthogonality relations}

The biorthogonality relations between functions $\psi_{n l}(x), l=0, \ldots, n-1$ follow from (2.1):

$$
\int_{-\infty}^{+\infty} \psi_{n l}(x) \psi_{n l^{\prime}}(x) d x=0, \quad l+l^{\prime} \leqslant n-1 .
$$

The biorthogonality relations between $\psi_{n 0}(x)$ and $\psi_{n}(x ; k)$ can be derived with the help of (2.3), (2.7) and (2.9):

$$
\int_{-\infty}^{+\infty} \psi_{n 0}(x)\left[k^{n} \psi_{n}(x ; k)\right] d x=i \int_{-\infty}^{+\infty} \psi_{n 0}(x)\left(-\partial+\frac{n}{x-z}\right)\left[k^{n-1} \psi_{n-1}(x ; k)\right] d x
$$




$$
\begin{aligned}
= & -\left.i \psi_{n 0}(x)\left[k^{n-1} \psi_{n-1}(x ; k)\right]\right|_{-\infty} ^{+\infty} \\
& +i \int_{-\infty}^{+\infty}\left[q_{n}^{-} \psi_{n 0}(x)\right]\left[k^{n-1} \psi_{n-1}(x ; k)\right] d x=0, \quad n=1,2,3, \ldots
\end{aligned}
$$

The biorthogonality relations between normalizable associated functions $\psi_{n l}(x)$ and $\psi_{n}(x ; k)$ can be derived in the same way with the help of (2.4) by induction,

$$
\begin{aligned}
\int_{-\infty}^{+\infty} & \psi_{n l}(x)\left[k^{n} \psi_{n}(x ; k)\right] d x=i \int_{-\infty}^{+\infty} \psi_{n l}(x)\left(-\partial+\frac{n}{x-z}\right)\left[k^{n-1} \psi_{n-1}(x ; k)\right] d x \\
& =-\left.i \psi_{n l}(x)\left[k^{n-1} \psi_{n-1}(x ; k)\right]\right|_{-\infty} ^{+\infty}+i \int_{-\infty}^{+\infty}\left[q_{n}^{-} \psi_{n l}(x)\right]\left[k^{n-1} \psi_{n-1}(x ; k)\right] d x \\
& =\int_{-\infty}^{+\infty} \psi_{n-1, l-1}(x)\left[k^{n-1} \psi_{n-1}(x ; k)\right] d x=\ldots \\
& =\int_{-\infty}^{+\infty} \psi_{n-l, 0}(x)\left[k^{n-l} \psi_{n-l}(x ; k)\right] d x=0, \quad l=1, \ldots,\left[\frac{n-1}{2}\right] .
\end{aligned}
$$

The biorthogonality relations between non-normalizable formal associated functions $\psi_{n l}(x)$, $l=[(n+1) / 2], \ldots, n-1$ and $\psi_{n}(x ; k)$,

$$
\int_{-\infty}^{+\infty} \psi_{n l}(x)\left[k^{n} \psi_{n}(x ; k)\right] d x=0, \quad l=\left[\frac{n+1}{2}\right], \ldots, n-1,
$$

can be derived with the help of (2.1), (2.6), the Jordan lemma and the relation 4.2.7.17 from [28] as follows,

$$
\begin{aligned}
& \int_{-\infty}^{+\infty} \psi_{n l}(x)\left[k^{n} \psi_{n}(x ; k)\right] d x \\
&=(-i)^{n} \frac{(2 n-2 l-1) ! !}{2 \pi(2 l) ! !} \sum_{m=0}^{2 l-n} \frac{i^{m}(n+m) !}{2^{m} m !(n-m) !} k^{n-m} \int_{-\infty}^{+\infty}(x-z)^{2 l-n-m} e^{i k x} d x \\
& \quad+(-i)^{n} \frac{(2 n-2 l-1) ! !}{2 \pi(2 l) ! !} \sum_{m=2 l-n+1}^{n} \frac{i^{m}(n+m) !}{2^{m} m !(n-m) !} k^{n-m} \int_{-\infty}^{+\infty} \frac{e^{i k x} d x}{(x-z)^{n-2 l+m}} \\
&=(-i)^{n} \frac{(2 n-2 l-1) ! !}{(2 l) ! !} \sum_{m=0}^{2 l-n} \frac{i^{m}(n+m) !}{2^{m} m !(n-m) !} k^{n-m}\left(-i \frac{d}{d k}-z\right)^{2 l-n-m} \delta(k) \\
& \quad+\operatorname{sign}(\operatorname{Im} z) \theta(\operatorname{sign}(\operatorname{Im} z) k)(-i)^{n} \frac{(2 n-2 l-1) ! !}{(2 l) ! !} e^{i k z} \\
& \quad \times \quad \sum_{m=2 l-n+1}^{n} \frac{i^{n-2 l+2 m}(n+m) !}{\left.2^{m} m-m\right) !(n-2 l+m-1) !} k^{2 n-2 l-1} \\
&=(-i)^{n} \frac{(2 n-2 l-1) ! !}{(2 l) ! !} \sum_{m=0}^{2 l-n} \frac{i^{m}(n+m) !}{2^{m} m !(n-m) !} k^{n-m}\left(-i \frac{d}{d k}-z\right)^{(n-m)-2(n-l)} \delta(k) \\
&+\operatorname{sign}(\operatorname{Im} z) \theta(\operatorname{sign}(\operatorname{Im} z) k)(-1)^{l} 2^{n} \frac{(2 n-2 l-1) ! !(2 l+1) ! !}{(2 n) ! !} k^{2 n-2 l-1} e^{i k z} \\
& \times \sum_{m=2 l-n+1}^{n} \frac{(-1)^{m}}{2^{m}} C_{n}^{m} C_{m+n}^{2 l+1}=0,
\end{aligned}
$$

where

$$
\theta(t)= \begin{cases}1, & t \geqslant 0 \\ 0, & t<0\end{cases}
$$


The formal associated functions $\psi_{n l}(x), l=n, n+1, n+2, \ldots$ are not contained in the resolutions of identity (see Section 2.3), but it is interesting that one can write the biorthogonality relations for these functions with $\psi_{n}(x ; k)$ as well,

$$
\begin{aligned}
\int_{-\infty}^{+\infty} & \psi_{n l}(x)\left[e^{-i k z} k^{n} \psi_{n}(x ; k)\right] d x \\
& =\frac{(-1)^{l} i^{n}}{2 \pi(2 l) ! !(2 l-2 n-1) ! !} e^{-i k z} \sum_{m=0}^{n} \frac{i^{m}(n+m) !}{2^{m} m !(n-m) !} k^{n-m} \int_{-\infty}^{+\infty}(x-z)^{2 l-n-m} e^{i k x} d x \\
& =\frac{(-1)^{l} i^{n}}{(2 l) ! !(2 l-2 n-1) ! !} e^{-i k z} \sum_{m=0}^{n} \frac{i^{m}(n+m) !}{2^{m} m !(n-m) !} k^{n-m}\left(-i \frac{d}{d k}-z\right)^{2 l-n-m} \delta(k) \\
& =\frac{(-1)^{n}}{(2 l) ! !(2 l-2 n-1) ! !} e^{-i k z} \sum_{m=0}^{n} \frac{(-1)^{m}(n+m) !}{2^{m} m !(n-m) !} k^{n-m}\left(\frac{d}{d k}-i z\right)^{2 l-n-m}\left[e^{i k z} \delta(k)\right] \\
& =\frac{(-1)^{n}}{(2 l) ! !(2 l-2 n-1) ! !} \sum_{m=0}^{n} \frac{(-1)^{m}(n+m) !}{2^{m} m !(n-m) !} k^{n-m} \delta^{(2 l-n-m)}(k) \\
& =\frac{1}{(2 l) ! !(2 l-2 n-1) ! !(2 l-2 n) !} \delta^{(2 l-2 n)}(k) \sum_{m=0}^{n} \frac{(n+m) !(2 l-n-m) !}{2^{m} m !(n-m) !} \\
& =\frac{1}{(2 l-2 n) !} \delta^{(2 l-2 n)}(k), \quad l=n, n+1, n+2, \ldots,
\end{aligned}
$$

where we have used (2.1), (2.6) and the relation

$$
\begin{aligned}
\sum_{m=0}^{n} & \frac{(n+m) !(s-m) !}{2^{m} m !(n-m) !}=\sum_{m=0}^{n} \frac{(n-m+2 m)(n-1+m) !(s-m) !}{2^{m} m !(n-m) !} \\
& =\sum_{m=0}^{n-1} \frac{(n-1+m) !(s-m) !}{2^{m} m !(n-1-m) !}+\sum_{m=1}^{n} \frac{(n-1+m) !(s-m) !}{2^{m-1}(m-1) !(n-m) !} \\
& =\sum_{m=0}^{n-1} \frac{(n-1+m) !(s-m) !}{2^{m} m !(n-1-m) !}+\sum_{m=0}^{n-1} \frac{(n+m) !(s-1-m) !}{2^{m} m !(n-1-m) !} \\
& =(s+n) \sum_{m=0}^{n-1} \frac{(n-1+m) !(s-1-m) !}{2^{m} m !(n-1-m) !}=\cdots=(s+n) \cdots(s-n+2)(s-n) ! \\
& =(s-n-1) ! !(s+n) ! !, \quad s \geqslant n .
\end{aligned}
$$

At last, the biorthogonality relations between eigenfunctions for continuous spectrum of the Hamiltonian $h_{n}$ are proved in [13] and take the following form:

$$
\int_{-\infty}^{+\infty}\left[k^{n} \psi_{n}(x ; k)\right]\left[\left(k^{\prime}\right)^{n} \psi_{n}\left(x ;-k^{\prime}\right)\right] d x=\left(k^{\prime}\right)^{2 n} \delta\left(k-k^{\prime}\right) .
$$

Let us notice that (2.13)-(2.15) contain (2.12) for $l=0, \ldots, n-1, l^{\prime}=0$ and (2.17) contains (2.12) for $l=l^{\prime}=0$ due to (2.11). The relations (2.16) can be derived with the help of differentiation from (2.17) also in view of (2.11).

\subsection{Resolutions of identity}

The initial resolution of identity constructed from $\psi_{n}(x ; k)$ holds [13],

$$
\delta\left(x-x^{\prime}\right)=\int_{\mathcal{L}} \psi_{n}(x ; k) \psi_{n}\left(x^{\prime} ;-k\right) d k,
$$


where $\mathcal{L}$ is an integration path in complex $k$ plane, obtained from the real axis by its deformation near the point $k=0$ upwards or downwards (the direction of this deformation is of no difference since the residue of the integrand for the point $k=0$ is equal zero in view of (2.6) and (2.10)) and the direction of $\mathcal{L}$ is specified from $-\infty$ to $+\infty$. This resolution of identity is valid for test functions belonging to $C L_{\gamma} \equiv C_{\mathbb{R}}^{\infty} \cap L_{2}\left(\mathbb{R} ;(1+|x|)^{\gamma}\right), \gamma>-1$ as well as for some bounded and even slowly increasing test functions (more details are presented in [13]) and, in particular, for eigenfunctions $\psi_{n}(x ; k)$ and for the associated function $(2.2)$.

One can rearrange the resolution of identity (2.18) for any $\varepsilon>0$ to the forms

$$
\begin{aligned}
& \delta\left(x-x^{\prime}\right)=\left(\int_{-\infty}^{-\varepsilon}+\int_{\varepsilon}^{+\infty}\right) \psi_{n}(x ; k) \psi_{n}\left(x^{\prime} ;-k\right) d k \\
& \quad+\left.\sum_{l=0}^{n-1}\left(\frac{x^{\prime}-z}{x-z}\right)^{l} \frac{\psi_{n-l-1}(x ; k) \psi_{n-l}\left(x^{\prime} ;-k\right)}{i(x-z)}\right|_{-\varepsilon} ^{\varepsilon}+\left(\frac{x^{\prime}-z}{x-z}\right)^{n} \frac{\sin \varepsilon\left(x-x^{\prime}\right)}{\pi\left(x-x^{\prime}\right)} \\
& \equiv\left(\int_{-\infty}^{-\varepsilon}+\int_{\varepsilon}^{+\infty}\right) \psi_{n}(x ; k) \psi_{n}\left(x^{\prime} ;-k\right) d k+\frac{\sin \varepsilon\left(x-x^{\prime}\right)}{\pi\left(x-x^{\prime}\right)} \\
& \quad-\frac{\cos \varepsilon\left(x-x^{\prime}\right)}{2 \pi \varepsilon(x-z)\left(x^{\prime}-z\right)} \sum_{l=0}^{n-1} \frac{(-1 / 4)^{l}}{\varepsilon^{2 l}\left(x^{\prime}-z\right)^{2 l}} \sum_{m=0}^{\min \{2 l, n-1\}} C_{2 l+1, m, n} \frac{(n+2 l+1-m) !}{(n-1-m) !}\left(\frac{x^{\prime}-z}{x-z}\right)^{m} \\
& \quad+\frac{\sin \varepsilon\left(x-x^{\prime}\right)}{\pi(x-z)} \sum_{l=1}^{n-1} \frac{(-1 / 4)^{l}}{\varepsilon^{2 l}\left(x^{\prime}-z\right)^{2 l}} \sum_{m=0}^{\min \{2 l-1, n-1\}} C_{2 l, m, n} \frac{(n+2 l-m) !}{(n-1-m) !}\left(\frac{x^{\prime}-z}{x-z}\right)^{m},(2.19) \\
& C_{l m n}=\frac{1}{l} \sum_{j=0}^{m}(-1)^{j} C_{l}^{j} C_{n-m-1+2 j}^{l-1}, \quad l=1, \ldots, 2 n-1, \quad m=0, \ldots, \min \{l-1, n-1\} \\
& n=1,2,3, \ldots, \quad l=1, \quad m
\end{aligned}
$$

(cf. with (68) in [2]) and, consequently, to the form

$$
\begin{aligned}
\delta(x- & \left.x^{\prime}\right)=\lim _{\varepsilon \downarrow 0}\left\{\left(\int_{-\infty}^{-\varepsilon}+\int_{\varepsilon}^{+\infty}\right) \psi_{n}(x ; k) \psi_{n}\left(x^{\prime} ;-k\right) d k+\frac{\sin \varepsilon\left(x-x^{\prime}\right)}{\pi\left(x-x^{\prime}\right)}\right. \\
& -\frac{\cos \varepsilon\left(x-x^{\prime}\right)}{2 \pi \varepsilon(x-z)\left(x^{\prime}-z\right)} \sum_{l=0}^{n-1} \frac{(-1 / 4)^{l}}{\varepsilon^{2 l}\left(x^{\prime}-z\right)^{2 l}} \sum_{m=0}^{\min \{2 l, n-1\}} C_{2 l+1, m, n} \frac{(n+2 l+1-m) !}{(n-1-m) !}\left(\frac{x^{\prime}-z}{x-z}\right)^{m} \\
& \left.+\frac{\sin \varepsilon\left(x-x^{\prime}\right)}{\pi(x-z)} \sum_{l=1}^{n-1} \frac{(-1 / 4)^{l}}{\varepsilon^{2 l}\left(x^{\prime}-z\right)^{2 l}} \sum_{m=0}^{\min \{2 l-1, n-1\}} C_{2 l, m, n} \frac{(n+2 l-m) !}{(n-1-m) !}\left(\frac{x^{\prime}-z}{x-z}\right)^{m}\right\},(2.20)
\end{aligned}
$$

where the prime' at the limit symbol emphasizes that this limit is regarded as a limit in the space of distributions (see details in [13]).

From (2.19) and (2.20) one can try to derive various reduced resolutions of identity similar to the resolutions (69) and (70) of [2], which correspond to the partial case $n=1$, or to the resolutions (15) and (16) of [29]. In particular, by virtue of Lemma 3.7 from [13], for test functions from $C L_{\gamma}, \gamma>-1$ the resolution (2.20) can be reduced to the form

$$
\begin{aligned}
& \delta\left(x-x^{\prime}\right)=\lim _{\varepsilon \downarrow 0}\left\{\left(\int_{-\infty}^{-\varepsilon}+\int_{\varepsilon}^{+\infty}\right) \psi_{n}(x ; k) \psi_{n}\left(x^{\prime} ;-k\right) d k\right. \\
& \quad-\frac{\cos \varepsilon\left(x-x^{\prime}\right)}{2 \pi \varepsilon(x-z)\left(x^{\prime}-z\right)} \sum_{l=0}^{n-1} \frac{(-1 / 4)^{l}}{\varepsilon^{2 l}\left(x^{\prime}-z\right)^{2 l}} \sum_{m=0}^{\min \{2 l, n-1\}} C_{2 l+1, m, n} \frac{(n+2 l+1-m) !}{(n-1-m) !}\left(\frac{x^{\prime}-z}{x-z}\right)^{m}
\end{aligned}
$$




$$
\left.+\frac{\sin \varepsilon\left(x-x^{\prime}\right)}{\pi(x-z)} \sum_{l=1}^{n-1} \frac{(-1 / 4)^{l}}{\varepsilon^{2 l}\left(x^{\prime}-z\right)^{2 l}} \sum_{m=0}^{\min \{2 l-1, n-1\}} C_{2 l, m, n} \frac{(n+2 l-m) !}{(n-1-m) !}\left(\frac{x^{\prime}-z}{x-z}\right)^{m}\right\} .
$$

More reduced resolutions of identity for the partial case $n=2$ are presented in the forthcoming Section 2.4 .

There is another way to transform the resolution (2.18) as well. This way was used for obtaining of the resolution of identity (26) from [29]. The integral from the right-hand part of (2.18) is understood [13] as follows:

$$
\int_{\mathcal{L}} \psi_{n}(x ; k) \psi_{n}\left(x^{\prime} ;-k\right) d k=\lim _{A \rightarrow+\infty}^{\prime} \int_{\mathcal{L}(A)} \psi_{n}(x ; k) \psi_{n}\left(x^{\prime} ;-k\right) d k,
$$

where $\mathcal{L}(A)$ is a path in complex $k$ plane, made of the segment $[-A, A]$ by its deformation near the point $k=0$ upwards or downwards (the direction of this deformation is of no difference as well as in the case with $\mathcal{L}$ ) and the direction of $\mathcal{L}(A)$ is specified from $-A$ to $A$. When using the facts that the integral in the right-hand part of (2.22) is a standard integral (not a distribution) and that $k^{2 n} \psi_{n}(x ; k) \psi_{n}\left(x^{\prime} ;-k\right)$ is an entire function of $k$ (see $\left.(2.6)\right)$ as well as employing the Leibniz formula and the formulae (2.10) and (2.11), we can transform (2.22) as follows,

$$
\begin{aligned}
\int_{\mathcal{L}} \psi_{n}(x ; k) \psi_{n}\left(x^{\prime} ;-k\right) d k=\lim _{A \rightarrow+\infty}^{\prime} \lim _{\varepsilon \downarrow 0}\left\{\left(\int_{-A}^{-\varepsilon}+\int_{\varepsilon}^{A}\right) \psi_{n}(x ; k) \psi_{n}\left(x^{\prime} ;-k\right) d k\right. \\
\left.+\left.\sum_{j=0}^{2 n-1} \frac{1}{j !} \frac{\partial^{j}}{\partial k^{j}}\left[k^{2 n} \psi_{n}(x ; k) \psi_{n}\left(x^{\prime} ;-k\right)\right]\right|_{k=0} \int_{\mathcal{L}(\varepsilon)} \frac{d k}{k^{2 n-j}}\right\} \\
=\lim _{A \rightarrow+\infty} \lim _{\varepsilon \downarrow 0}\left\{\left(\int_{-A}^{-\varepsilon}+\int_{\varepsilon}^{A}\right) \psi_{n}(x ; k) \psi_{n}\left(x^{\prime} ;-k\right) d k\right. \\
\left.-2(-1)^{n} \sum_{l=0}^{n-1} \frac{1}{(2 n-2 l-1) \varepsilon^{2 n-2 l-1}} \sum_{m=0}^{l} \psi_{n m}(x) \psi_{n, l-m}\left(x^{\prime}\right)\right\} \\
=\lim _{\varepsilon \downarrow 0}^{*}\left\{\left(\int_{-\infty}^{-\varepsilon}+\int_{\varepsilon}^{+\infty}\right) \psi_{n}(x ; k) \psi_{n}\left(x^{\prime} ;-k\right) d k\right. \\
\left.\quad-2(-1)^{n} \sum_{l=0}^{n-1} \frac{1}{(2 n-2 l-1) \varepsilon^{2 n-2 l-1}} \sum_{m=0}^{l} \psi_{n m}(x) \psi_{n, l-m}\left(x^{\prime}\right)\right\},
\end{aligned}
$$

where the latter equality is considered as a definition and the limit $\lim _{\varepsilon \downarrow 0}$ is regarded as point-wise one (not as a limit in a function space).

Let us show that the terms outside the integral in the last line of (2.23) can be rearranged as follows,

$$
\begin{aligned}
& -2(-1)^{n} \sum_{l=0}^{n-1} \frac{1}{(2 n-2 l-1) \varepsilon^{2 n-2 l-1}} \sum_{m=0}^{l} \psi_{n m}(x) \psi_{n, l-m}\left(x^{\prime}\right) \\
& =\sum_{l=0}^{n-1} \psi_{n l}(x ; \varepsilon) \psi_{n, n-1-l}\left(x^{\prime} ; \varepsilon\right),
\end{aligned}
$$

where $\psi_{n l}(x ; \varepsilon), l=0, \ldots, n-1$ is the chain of eigenfunction and associated functions (formal for $l=[(n+1) / 2], \ldots, n-1)$ of the Hamiltonian $h_{n}$ for the eigenvalue $E=0$ of the form

$$
\psi_{n l}(x ; \varepsilon)=\sum_{j=0}^{l} \alpha_{j}(\varepsilon) \psi_{n, l-j}(x), \quad l=0, \ldots, n-1,
$$




$$
h_{n} \psi_{n 0}(x ; \varepsilon)=0, \quad h_{n} \psi_{n l}(x ; \varepsilon)=\psi_{n, l-1}(x ; \varepsilon), \quad l=1, \ldots, n-1
$$

with $\alpha_{j}(\varepsilon), j=0, \ldots, n-1$ being unknown coefficients which will be found below. Using (2.24) and $(2.25)$, it is easy to check that $(2.24)$ is valid iff the coefficients $\alpha_{j}(x)$ satisfy the following system,

$$
\sum_{j=0}^{l} \alpha_{j}(\varepsilon) \alpha_{l-j}(\varepsilon)=-\frac{2(-1)^{n}}{(2 l+1) \varepsilon^{2 l+1}}, \quad l=0, \ldots, n-1 .
$$

After the redefinition

$$
\alpha_{j}(\varepsilon)=\frac{\sqrt{2} i^{n+1}}{\varepsilon^{2 j} \sqrt{\varepsilon}} \beta_{j}, \quad j=0, \ldots, n-1,
$$

where $\beta_{j}, j=0, \ldots, n-1$ are new unknown coefficients, the system (2.26) takes the form

$$
\sum_{j=0}^{l} \beta_{j} \beta_{l-j}=\frac{1}{2 l+1}, \quad l=0, \ldots, n-1 .
$$

The general solution of the latter system can be found in the recurrent form,

$$
\beta_{0}= \pm 1, \quad \beta_{1}=\frac{1}{6 \beta_{0}}, \quad \beta_{l}=\frac{1}{2 \beta_{0}}\left(\frac{1}{2 l+1}-\sum_{j=1}^{l-1} \beta_{j} \beta_{l-j}\right), \quad l=2, \ldots, n-1 .
$$

The first terms of the sequence $\beta_{j}, j=0, \ldots, n-1$ in the case $\beta_{0}=1$ are the following ones,

$$
\beta_{0}=1, \quad \beta_{1}=\frac{1}{6}, \quad \beta_{2}=\frac{31}{360}, \quad \beta_{3}=\frac{863}{15120}, \quad \beta_{4}=\frac{76813}{1814400}, \quad \ldots
$$

Thus, we can choose the functions $\psi_{n l}(x ; \varepsilon)$ in the form,

$$
\psi_{n l}(x ; \varepsilon)=i^{n+1} \sqrt{\frac{2}{\varepsilon}} \sum_{j=0}^{l} \frac{\beta_{j}}{\varepsilon^{2 j}} \psi_{n, l-j}(x), \quad l=0, \ldots, n-1
$$

and the resolution of identity holds,

$$
\begin{aligned}
\delta\left(x-x^{\prime}\right)= & \lim _{\varepsilon \downarrow 0} *\left\{\left(\int_{-\infty}^{-\varepsilon}+\int_{\varepsilon}^{+\infty}\right) \psi_{n}(x ; k) \psi_{n}\left(x^{\prime} ;-k\right) d k\right. \\
& \left.+\sum_{l=0}^{n-1} \psi_{n l}(x ; \varepsilon) \psi_{n, n-1-l}\left(x^{\prime} ; \varepsilon\right)\right\},
\end{aligned}
$$

(cf. with (69) from [2] for the case $n=1$ ). Moreover, this resolution is equivalent to (2.18), i.e. it is valid for all test functions for which (2.18) is valid (cf. with the analogous results in Section 3 and in [29]).

The resolution of identity (2.28) contains all $n$ functions from the chain $\psi_{n l}(x ; \varepsilon), l=$ $0, \ldots, n-1$ and in this resolution for the eigenvalue $E=0$ there are no other eigen- or associated functions of the Hamiltonian $h_{n}$. The order of the pole $k=0$ for the Green function

$$
\begin{aligned}
& G_{n}\left(x, x^{\prime} ; E\right)=\left.\left[\frac{\pi i}{k} \psi_{n}\left(x_{>} ; k\right) \psi_{n}\left(x_{<} ;-k\right)\right]\right|_{k=\sqrt{E}}, \\
& x_{>}=\max \left\{x, x^{\prime}\right\}, \quad x_{<}=\left\{x, x^{\prime}\right\}, \quad \operatorname{Im} \sqrt{E} \geqslant 0, \quad\left(h_{n}-E\right) G_{n}=\delta\left(x-x^{\prime}\right)
\end{aligned}
$$


considered as a function of $k=\sqrt{E}$ is equal to $2 n+1$ in view of (2.6), and the exceptional point $E=0$ coincides with the branch point of this Green function as a function of $E$. One can consider this pole of the order $2 n+1$ as a result of confluence of the pole of the order $n$ for the Green function as a function of $E=k^{2}$ and of the factor $k \equiv \sqrt{E}$ from the denominator of the Green function (see (2.29)). Thus, the number $n$ of linearly independent eigen- and (formal) associated functions of the Hamiltonian $h_{n}$ incorporated in the resolution of identity (2.28) for the eigenvalue $E=0$ (exceptional point) is equal to the order of the "pole" $E=0$ for the Green function $G_{n}\left(x, x^{\prime} ; E\right)$ in the sense elucidated above or, more rigorously, the order of the pole of $G_{n}\left(x, x^{\prime} ; E\right)$ as a function of $k=\sqrt{E}$ is $2 n+1$ expressed in terms of the number $n$.

Let us notice that in view of $(2.27)$ the functions $\psi_{n l}(x ; \varepsilon), l=0, \ldots, n-1$ satisfy the same biorthogonality relations from Section 2.2 as the functions $\psi_{n l}(x), l=0, \ldots, n-1$.

\subsection{Example: case $n=2$}

For the Hamiltonian

$$
h_{2}=-\partial^{2}+\frac{6}{(x-z)^{2}}, \quad x \in \mathbb{R}, \quad \operatorname{Im} z \neq 0,
$$

there are continuous spectrum eigenfunctions

$$
\begin{aligned}
& \psi_{2}(x ; k)=\frac{1}{\sqrt{2 \pi}}\left[1-\frac{3}{i k(x-z)}-\frac{3}{k^{2}(x-z)^{2}}\right] e^{i k x}, \\
& h_{2} \psi_{2}(x ; k)=k^{2} \psi_{2}(x ; k), \quad k \in(-\infty, 0) \cup(0,+\infty)
\end{aligned}
$$

(see (2.6)) and also the normalizable eigenfunction $\psi_{20}(x)$ and the bounded associated function $\psi_{21}(x)$ on the level $E=0$,

$$
\psi_{20}(x)=-\frac{3}{\sqrt{2 \pi}(x-z)^{2}}, \quad \psi_{21}(x)=-\frac{1}{2 \sqrt{2 \pi}}, \quad h_{2} \psi_{20}=0, \quad h_{2} \psi_{21}=\psi_{20},
$$

(see (2.1) and (2.2)). In this case at the point $k=0$ there is a fifth order pole in the Green function $G_{2}\left(x, x^{\prime} ; E\right)$ considered as a function of $k=\sqrt{E}$ (see (2.29) and (2.30)) and thereby the exceptional point $E=0$ of the spectrum of the Hamiltonian $h_{2}$ coincides with the branch point for the Green function as a function of $E$.

In the case under consideration the biorthogonality relations (2.12)-(2.15) and (2.17) take the form,

$$
\begin{aligned}
& \int_{-\infty}^{+\infty} \psi_{20}^{2}(x) d x=0, \quad \int_{-\infty}^{+\infty} \psi_{20}(x)\left[k^{2} \psi_{2}(x ; k)\right] d x=0 \\
& \int_{-\infty}^{+\infty} \psi_{20}(x) \psi_{21}(x) d x=0, \quad \int_{-\infty}^{+\infty} \psi_{21}(x)\left[k^{2} \psi_{2}(x ; k)\right] d x=0 \\
& \int_{-\infty}^{+\infty}\left[k^{2} \psi_{2}(x ; k)\right]\left[\left(k^{\prime}\right)^{2} \psi_{2}\left(x ;-k^{\prime}\right)\right] d x=\left(k^{\prime}\right)^{4} \delta\left(k-k^{\prime}\right),
\end{aligned}
$$

where (2.31) are included in (2.33) due to the equality

$$
\psi_{20}(x)=\lim _{k \rightarrow 0}\left[k^{2} \psi_{2}(x ; k)\right]
$$

(see (2.11)) and (2.32) can be derived from (2.33) in view of (2.34) and of the equality

$$
\psi_{21}(x)=\frac{1}{2} \lim _{k \rightarrow 0} \frac{\partial^{2}}{\partial k^{2}}\left[e^{-i k z} k^{2} \psi_{2}(x ; k)\right]
$$

(see (2.11) as well). 
It is straightforward to check that the resolution of identity (2.19) (which is valid for test functions from $C L_{\gamma}, \gamma>-1$ as well as for some bounded and even slowly increasing test functions, see Section 2.3), can be rewritten in the form

$$
\begin{aligned}
\delta(x- & \left.x^{\prime}\right)=\left(\int_{-\infty}^{-\varepsilon}+\int_{\varepsilon}^{+\infty}\right) \psi_{2}(x ; k) \psi_{2}\left(x^{\prime} ;-k\right) d k \\
& +\left[\psi_{20}(x ; \varepsilon) \psi_{21}\left(x^{\prime} ; \varepsilon\right)+\psi_{21}(x ; \varepsilon) \psi_{20}\left(x^{\prime} ; \varepsilon\right)\right]+\frac{\sin \varepsilon\left(x-x^{\prime}\right)}{\pi\left(x-x^{\prime}\right)}+\frac{6 \sin ^{2} \frac{\varepsilon}{2}\left(x-x^{\prime}\right)}{\pi \varepsilon(x-z)\left(x^{\prime}-z\right)} \\
& +\frac{12\left(x-x^{\prime}\right) \sin ^{2} \frac{\varepsilon}{4}\left(x-x^{\prime}\right) \sin \frac{\varepsilon}{2}\left(x-x^{\prime}\right)}{\pi \varepsilon^{2}(x-z)^{2}\left(x^{\prime}-z\right)^{2}}+\frac{3\left[\varepsilon\left(x-x^{\prime}\right)-2 \sin \frac{\varepsilon}{2}\left(x-x^{\prime}\right)\right]^{2}}{2 \pi \varepsilon^{3}(x-z)^{2}\left(x^{\prime}-z\right)^{2}}
\end{aligned}
$$

where the eigenfunction $\psi_{20}(x ; \varepsilon)$ and the associated function $\psi_{21}(x ; \varepsilon)$ of the Hamiltonian $h_{2}$ read

$$
\begin{aligned}
& \psi_{20}(x ; \varepsilon)=-i \sqrt{\frac{2}{\varepsilon}} \psi_{20}(x) \equiv \frac{3 i}{\sqrt{\pi \varepsilon}(x-z)^{2}}, \\
& \psi_{21}(x ; \varepsilon)=-i \sqrt{\frac{2}{\varepsilon}}\left[\psi_{21}(x)+\frac{1}{6 \varepsilon^{2}} \psi_{20}(x)\right] \equiv \frac{i}{2 \sqrt{\pi \varepsilon}}\left[1+\frac{1}{\varepsilon^{2}(x-z)^{2}}\right], \\
& h_{2} \psi_{20}(x ; \varepsilon)=0, \quad h_{2} \psi_{21}(x ; \varepsilon)=\psi_{20}(x ; \varepsilon) .
\end{aligned}
$$

The eigenfunction $\psi_{20}(x ; \varepsilon)$ and the associated function $\psi_{21}(x ; \varepsilon)$ obviously satisfy the biorthogonality relations similar to $(2.31)$ and $(2.32)$.

It is shown in [13] that the resolution of identity (2.35) can be reduced: a) for test functions from $C L_{\gamma}, \gamma>-1$ to the form

$$
\begin{aligned}
\delta(x- & \left.x^{\prime}\right)=\lim _{\varepsilon \downarrow 0}\left\{\left(\int_{-\infty}^{-\varepsilon}+\int_{\varepsilon}^{+\infty}\right) \psi_{2}(x ; k) \psi_{2}\left(x^{\prime} ;-k\right) d k\right. \\
& +\left[\psi_{20}(x ; \varepsilon) \psi_{21}\left(x^{\prime} ; \varepsilon\right)+\psi_{21}(x ; \varepsilon) \psi_{20}\left(x^{\prime} ; \varepsilon\right)\right]+\frac{6 \sin ^{2} \frac{\varepsilon}{2}\left(x-x^{\prime}\right)}{\pi \varepsilon(x-z)\left(x^{\prime}-z\right)} \\
& \left.+\frac{12\left(x-x^{\prime}\right) \sin ^{2} \frac{\varepsilon}{4}\left(x-x^{\prime}\right) \sin \frac{\varepsilon}{2}\left(x-x^{\prime}\right)}{\pi \varepsilon^{2}(x-z)^{2}\left(x^{\prime}-z\right)^{2}}+\frac{3\left[\varepsilon\left(x-x^{\prime}\right)-2 \sin \frac{\varepsilon}{2}\left(x-x^{\prime}\right)\right]^{2}}{2 \pi \varepsilon^{3}(x-z)^{2}\left(x^{\prime}-z\right)^{2}}\right\}
\end{aligned}
$$

identical to $(2.21)$, b) for test functions from $C L_{\gamma}, \gamma>1$ to the form

$$
\begin{aligned}
\delta(x- & \left.x^{\prime}\right)=\lim _{\varepsilon \downarrow 0}\left\{\left\{\left(\int_{-\infty}^{-\varepsilon}+\int_{\varepsilon}^{+\infty}\right) \psi_{2}(x ; k) \psi_{2}\left(x^{\prime} ;-k\right) d k\right.\right. \\
& +\left[\psi_{20}(x ; \varepsilon) \psi_{21}\left(x^{\prime} ; \varepsilon\right)+\psi_{21}(x ; \varepsilon) \psi_{20}\left(x^{\prime} ; \varepsilon\right)\right] \\
& \left.+\frac{12\left(x-x^{\prime}\right) \sin ^{2} \frac{\varepsilon}{4}\left(x-x^{\prime}\right) \sin \frac{\varepsilon}{2}\left(x-x^{\prime}\right)}{\pi \varepsilon^{2}(x-z)^{2}\left(x^{\prime}-z\right)^{2}}+\frac{3\left[\varepsilon\left(x-x^{\prime}\right)-2 \sin \frac{\varepsilon}{2}\left(x-x^{\prime}\right)\right]^{2}}{2 \pi \varepsilon^{3}(x-z)^{2}\left(x^{\prime}-z\right)^{2}}\right\}
\end{aligned}
$$

and c) for test functions from $C L_{\gamma}, \gamma>3$ to the form

$$
\begin{aligned}
\delta\left(x-x^{\prime}\right)= & \lim _{\varepsilon \downarrow 0}\left\{\left(\int_{-\infty}^{-\varepsilon}+\int_{\varepsilon}^{+\infty}\right) \psi_{2}(x ; k) \psi_{2}\left(x^{\prime} ;-k\right) d k\right. \\
& \left.+\psi_{20}(x ; \varepsilon) \psi_{21}\left(x^{\prime} ; \varepsilon\right)+\psi_{21}(x ; \varepsilon) \psi_{20}\left(x^{\prime} ; \varepsilon\right)\right\} .
\end{aligned}
$$


The latter of these resolutions of identity seems to have a more natural form than the previous ones, but the right-hand part of the latter resolution cannot reproduce the normalizable eigenfunction

$$
\psi_{20}(x) \notin C L_{\gamma} \equiv C_{\mathbb{R}}^{\infty} \cap L^{2}\left(\mathbb{R} ;(1+|x|)^{\gamma}\right), \quad \gamma>3
$$

because of the biorthogonality relations. With the help of the Jordan lemma one can check that

$$
\begin{aligned}
& \lim _{\varepsilon \downarrow 0} \int_{-\infty}^{+\infty} \frac{12\left(x-x^{\prime}\right) \sin ^{2} \frac{\varepsilon}{4}\left(x-x^{\prime}\right) \sin \frac{\varepsilon}{2}\left(x-x^{\prime}\right)}{\pi \varepsilon^{2}(x-z)^{2}\left(x^{\prime}-z\right)^{2}} \psi_{20}(x) d x \\
&= \lim _{\varepsilon \downarrow 0}\left\{\left[-\frac{3}{4} e^{ \pm i \varepsilon\left(z-x^{\prime}\right) / 2} \mp \frac{i}{8} \varepsilon\left(z-x^{\prime}\right) e^{ \pm i \varepsilon\left(z-x^{\prime}\right) / 2}+\frac{3}{2} e^{ \pm i \varepsilon\left(z-x^{\prime}\right)}\right.\right. \\
&\left.\left.\quad \pm \frac{i}{2} \varepsilon\left(z-x^{\prime}\right) e^{ \pm i \varepsilon\left(z-x^{\prime}\right)}\right] \psi_{20}\left(x^{\prime}\right)\right\}=\frac{3}{4} \psi_{20}\left(x^{\prime}\right)
\end{aligned}
$$

and

$$
\begin{aligned}
& \lim _{\varepsilon \downarrow 0} \int_{-\infty}^{+\infty} \frac{3\left[\varepsilon\left(x-x^{\prime}\right)-2 \sin \frac{\varepsilon}{2}\left(x-x^{\prime}\right)\right]^{2}}{2 \pi \varepsilon^{3}(x-z)^{2}\left(x^{\prime}-z\right)^{2}} \psi_{20}(x) d x \\
& \quad=\lim _{\varepsilon \downarrow 0}\left\{\left[\frac{3}{4} e^{ \pm i \varepsilon\left(z-x^{\prime}\right) / 2} \pm \frac{i}{8} \varepsilon\left(z-x^{\prime}\right) e^{ \pm i \varepsilon\left(z-x^{\prime}\right) / 2}-\frac{1}{2} e^{ \pm i \varepsilon\left(z-x^{\prime}\right)}\right] \psi_{20}\left(x^{\prime}\right)\right\}=\frac{1}{4} \psi_{20}\left(x^{\prime}\right),
\end{aligned}
$$

where the upper (lower) signs correspond to the case $\operatorname{Im} z>0(\operatorname{Im} z<0)$. Hence, just two last terms of the resolution of identity (2.37) and the corresponding terms in the resolutions of identity (2.35) and (2.36) give a chance to reproduce $\psi_{20}(x)$ by these resolutions (cf. with the analogous results in Section 6.1 of [2], in Section 2 of [29] and in Section 3 of the present paper). It is interesting that contributions of these terms in the resolution of identity are (see Remark 3.4 in [13]) singular discontinuous functionals whose supports consist of the only element which is the infinity (cf. with the analogous comments in Section 2 of [29] and in Section 3 of the present paper).

In the case under consideration the resolution of identity (2.28) takes the following form,

$$
\begin{aligned}
\delta\left(x-x^{\prime}\right)= & \lim _{\varepsilon \downarrow 0} *\left\{\left(\int_{-\infty}^{-\varepsilon}+\int_{\varepsilon}^{+\infty}\right) \psi_{2}(x ; k) \psi_{2}\left(x^{\prime} ;-k\right) d k\right. \\
& \left.+\psi_{20}(x ; \varepsilon) \psi_{21}\left(x^{\prime} ; \varepsilon\right)+\psi_{21}(x ; \varepsilon) \psi_{20}\left(x^{\prime} ; \varepsilon\right)\right\}
\end{aligned}
$$

(cf. with (2.38)) and it is valid for test functions from $C L_{\gamma}, \gamma>-1$ as well as for some bounded and even slowly increasing test functions (see Section 2.3).

\section{Resolutions of identity for the model Hamiltonian with exceptional point inside of the continuous spectrum}

For the Hamiltonian

$$
\begin{aligned}
& h=-\partial^{2}+16 \alpha^{2} \frac{\alpha(x-z) \sin 2 \alpha x+2 \cos ^{2} \alpha x}{[\sin 2 \alpha x+2 \alpha(x-z)]^{2}}, \\
& x \in \mathbb{R}, \quad \partial \equiv \frac{d}{d x}, \quad \alpha>0, \quad \operatorname{Im} z \neq 0
\end{aligned}
$$


there are [2] continuous spectrum eigenfunctions

$$
\begin{aligned}
& \psi(x ; k)=\frac{1}{\sqrt{2 \pi}}\left[1+\frac{i k}{k^{2}-\alpha^{2}} \frac{W^{\prime}(x)}{W(x)}-\frac{1}{2\left(k^{2}-\alpha^{2}\right)} \frac{W^{\prime \prime}(x)}{W(x)}\right] e^{i k x}, \\
& W(x)=\sin 2 \alpha x+2 \alpha(x-z), \\
& h \psi(x ; k)=k^{2} \psi(x ; k), \quad k \in(-\infty,-\alpha) \cup(-\alpha, \alpha) \cup(\alpha,+\infty) .
\end{aligned}
$$

As well for the level $E=\alpha^{2}$ there is the normalizable eigenfunction $\psi_{0}(x)$ and the bounded associated function ${ }^{1} \psi_{1}(x)$,

$$
\begin{aligned}
& \psi_{0}(x)=\frac{(2 \alpha)^{3 / 2} \cos \alpha x}{\sin 2 \alpha x+2 \alpha(x-z)}, \quad \psi_{1}(x)=\frac{2 \alpha(x-z) \sin \alpha x+\cos \alpha x}{\sqrt{2 \alpha}[\sin 2 \alpha x+2 \alpha(x-z)]}, \\
& \psi_{1}(x)=\frac{i}{2 \sqrt{2 \alpha}}\left[e^{-i \alpha x}-e^{i \alpha x}\right]+O\left(\frac{1}{x}\right), \quad x \rightarrow \pm \infty
\end{aligned}
$$

such that

$$
h \psi_{0}=\alpha^{2} \psi_{0}, \quad\left(h-\alpha^{2}\right) \psi_{1}=\psi_{0} .
$$

The exceptional point $E=\alpha^{2}$ is a pole for the Green function

$$
\begin{aligned}
& G\left(x, x^{\prime} ; E\right)=\left.\left[\frac{\pi i}{k} \psi_{n}\left(x_{>} ; k\right) \psi_{n}\left(x_{<} ;-k\right)\right]\right|_{k=\sqrt{E}}, \\
& x_{>}=\max \left\{x, x^{\prime}\right\}, \quad x_{<}=\left\{x, x^{\prime}\right\}, \quad \operatorname{Im} \sqrt{E} \geqslant 0, \quad(h-E) G=\delta\left(x-x^{\prime}\right) .
\end{aligned}
$$

This pole is a pole of second order, it is replicated on both sides of the cut $E>0$ and there are no other poles for $G\left(x, x^{\prime} ; E\right)$.

One can show [2] that the eigenfunctions and the associated function of $h$ obey the biorthogonality relations,

$$
\begin{aligned}
& \int_{-\infty}^{+\infty} \psi_{0}^{2}(x) d x=0, \quad \int_{-\infty}^{+\infty} \psi_{0}(x)\left[\left(k^{2}-\alpha^{2}\right) \psi(x ; k)\right] d x=0, \\
& \int_{-\infty}^{+\infty} \psi_{0}(x) \psi_{1}(x) d x=0, \quad \int_{-\infty}^{+\infty} \psi_{1}(x)\left[\left(k^{2}-\alpha^{2}\right) \psi(x ; k)\right] d x=0, \\
& \int_{-\infty}^{+\infty}\left[\left(k^{2}-\alpha^{2}\right) \psi(x ; k)\right]\left[\left(\left(k^{\prime}\right)^{2}-\alpha^{2}\right) \psi\left(x ;-k^{\prime}\right)\right] d x=\left(\left(k^{\prime}\right)^{2}-\alpha^{2}\right)^{2} \delta\left(k-k^{\prime}\right),
\end{aligned}
$$

where (3.3) are included in (3.5) due to the equality

$$
\psi_{0}(x)=\mp i \sqrt{\frac{\pi}{\alpha}} \lim _{k \rightarrow \pm \alpha}\left[\left(k^{2}-\alpha^{2}\right) \psi(x ; k)\right]
$$

and (3.4) follow from (3.5) in view of (3.6) and of the equality

$$
\psi_{1}(x)= \pm i \sqrt{\frac{\pi}{\alpha}} \lim _{k \rightarrow \mp \alpha}\left\{\frac{1}{2 k} \frac{\partial}{\partial k}\left[\left(k^{2}-\alpha^{2}\right)\right] \psi(x ; k)\right\}-\frac{1 \mp 2 i \alpha z}{4 \alpha^{2}} \psi_{0}(x) .
$$

The resolution of identity constructed from $\psi(x ; k)$ holds [13],

$$
\delta\left(x-x^{\prime}\right)=\int_{\mathcal{L}} \psi(x ; k) \psi\left(x^{\prime} ;-k\right) d k,
$$

\footnotetext{
${ }^{1}$ There is a misprint in the normalization of $\psi_{0}(x)$ and $\psi_{1}(x)$ in [2].
} 
where $\mathcal{L}$ is an integration path in complex $k$ plane, obtained from the real axis by its simultaneous deformation near the points $k=-\alpha$ and $k=\alpha$ upwards or downwards (the direction of this deformation is of no difference since for the points $k=-\alpha$ and $k=\alpha$ the sum of residues of the integrand is equal to zero). The direction of $\mathcal{L}$ is specified from $-\infty$ to $+\infty$. This resolution of identity is valid for test functions belonging to $C L_{\gamma} \equiv C_{\mathbb{R}}^{\infty} \cap L_{2}\left(\mathbb{R} ;(1+|x|)^{\gamma}\right), \gamma>-1$ as well as for some bounded and even slowly increasing test functions and, in particular, for eigenfunctions $\psi(x ; k)$ and for the associated function $\psi_{1}(x)$.

One can rearrange [13] the resolution of identity (3.7) for any $\varepsilon \in(0, \alpha)$ to the form

$$
\begin{aligned}
\delta\left(x-x^{\prime}\right)= & \left(\int_{-\infty}^{-\alpha-\varepsilon}+\int_{-\alpha+\varepsilon}^{\alpha-\varepsilon}+\int_{\alpha+\varepsilon}^{+\infty}\right) \psi(x ; k) \psi\left(x^{\prime} ;-k\right) d k \\
& +\frac{2}{\pi} \cos \alpha\left(x-x^{\prime}\right) \frac{\sin \varepsilon\left(x-x^{\prime}\right)}{x-x^{\prime}}-\frac{1}{\pi \alpha} \psi_{0}(x) \psi_{0}\left(x^{\prime}\right)\left[\frac{1}{\varepsilon}\left[1-2 \sin ^{2} \frac{\varepsilon}{2}\left(x-x^{\prime}\right)\right]\right. \\
& \left.-\frac{\varepsilon}{4 \alpha^{2}-\varepsilon^{2}} \cos 2 \alpha\left(x-x^{\prime}\right) \cos \varepsilon\left(x-x^{\prime}\right)-\frac{2 \alpha}{4 \alpha^{2}-\varepsilon^{2}} \sin 2 \alpha\left(x-x^{\prime}\right) \sin \varepsilon\left(x-x^{\prime}\right)\right] \\
& -\frac{1}{\pi}\left[\psi_{0}(x) \psi_{1}\left(x^{\prime}\right)+\psi_{1}(x) \psi_{0}\left(x^{\prime}\right)\right] \int_{2 \alpha-\varepsilon}^{2 \alpha+\varepsilon} \cos t\left(x-x^{\prime}\right) \frac{d t}{t}
\end{aligned}
$$

and, consequently, to the form

$$
\begin{aligned}
\delta\left(x-x^{\prime}\right)= & \lim _{\varepsilon \downarrow 0}\left\{\left(\int_{-\infty}^{-\alpha-\varepsilon}+\int_{-\alpha+\varepsilon}^{\alpha-\varepsilon}+\int_{\alpha+\varepsilon}^{+\infty}\right) \psi(x ; k) \psi\left(x^{\prime} ;-k\right) d k\right. \\
& +\frac{2}{\pi} \cos \alpha\left(x-x^{\prime}\right) \frac{\sin \varepsilon\left(x-x^{\prime}\right)}{x-x^{\prime}}-\frac{1}{\pi \alpha} \psi_{0}(x) \psi_{0}\left(x^{\prime}\right)\left[\frac{1}{\varepsilon}\left[1-2 \sin ^{2} \frac{\varepsilon}{2}\left(x-x^{\prime}\right)\right]\right. \\
& \left.-\frac{\varepsilon}{4 \alpha^{2}-\varepsilon^{2}} \cos 2 \alpha\left(x-x^{\prime}\right) \cos \varepsilon\left(x-x^{\prime}\right)-\frac{2 \alpha}{4 \alpha^{2}-\varepsilon^{2}} \sin 2 \alpha\left(x-x^{\prime}\right) \sin \varepsilon\left(x-x^{\prime}\right)\right] \\
& \left.-\frac{1}{\pi}\left[\psi_{0}(x) \psi_{1}\left(x^{\prime}\right)+\psi_{1}(x) \psi_{0}\left(x^{\prime}\right)\right] \int_{2 \alpha-\varepsilon}^{2 \alpha+\varepsilon} \cos t\left(x-x^{\prime}\right) \frac{d t}{t}\right\}
\end{aligned}
$$

where the prime' at the limit symbol emphasizes that this limit is regarded as a limit in the space of distributions.

One can reduce [13] the resolutions of identity (3.8) and (3.9) for test functions from $C L_{\gamma}$, $\gamma>-1$ to the form

$$
\begin{aligned}
\delta\left(x-x^{\prime}\right)= & \lim _{\varepsilon \downarrow 0}\left\{\left(\int_{-\infty}^{-\alpha-\varepsilon}+\int_{-\alpha+\varepsilon}^{\alpha-\varepsilon}+\int_{\alpha+\varepsilon}^{+\infty}\right) \psi(x ; k) \psi\left(x^{\prime} ;-k\right) d k\right. \\
& \left.-\frac{1}{\pi \varepsilon \alpha}\left[1-2 \sin ^{2} \frac{\varepsilon}{2}\left(x-x^{\prime}\right)\right] \psi_{0}(x) \psi_{0}\left(x^{\prime}\right)\right\}
\end{aligned}
$$

and for test functions from $C L_{\gamma}, \gamma>1$ to a more simple form

$$
\delta\left(x-x^{\prime}\right)=\lim _{\varepsilon \downarrow 0}\left\{\left(\int_{-\infty}^{-\alpha-\varepsilon}+\int_{-\alpha+\varepsilon}^{\alpha-\varepsilon}+\int_{\alpha+\varepsilon}^{+\infty}\right) \psi(x ; k) \psi\left(x^{\prime} ;-k\right) d k-\frac{1}{\pi \varepsilon \alpha} \psi_{0}(x) \psi_{0}\left(x^{\prime}\right)\right\} .
$$

The latter of these resolutions seems to have a more natural form than the previous ones, but it cannot reproduce the normalizable eigenfunction

$$
\psi_{0}(x) \notin C L_{\gamma} \equiv C_{\mathbb{R}}^{\infty} \cap L^{2}\left(\mathbb{R} ;(1+|x|)^{\gamma}\right), \quad \gamma>1
$$


because of the biorthogonality relations. With the help of (3.2), Lemma 4.8 from [13] and the Jordan lemma one can check that

$$
\begin{aligned}
\lim _{\varepsilon \downarrow 0} \int_{-\infty}^{+\infty} & {\left[\frac{2}{\pi \varepsilon \alpha} \sin ^{2} \frac{\varepsilon}{2}\left(x-x^{\prime}\right) \psi_{0}(x) \psi_{0}\left(x^{\prime}\right)\right] \psi_{0}(x) d x } \\
= & \lim _{\varepsilon \downarrow 0}\left\{\psi_{0}\left(x^{\prime}\right) \int_{-\infty}^{+\infty}\left[\frac{2}{\pi \varepsilon \alpha} \sin ^{2} \frac{\varepsilon}{2}\left(x-x^{\prime}\right)\right]\right. \\
& \left.\times\left[2 \alpha \frac{\cos ^{2} \alpha x}{(x-z)^{2}}-\frac{\sin 2 \alpha x[\sin 2 \alpha x+4 \alpha(x-z)]}{4 \alpha^{2}(x-z)^{2}} \psi_{0}^{2}(x)\right] d x\right\} \\
= & \lim _{\varepsilon \downarrow 0}\left\{\psi_{0}\left(x^{\prime}\right) \int_{-\infty}^{+\infty}\left[\frac{2}{\pi \varepsilon \alpha} \sin ^{2} \frac{\varepsilon}{2}\left(x-x^{\prime}\right)\right]\left[2 \alpha \frac{\cos ^{2} \alpha x}{(x-z)^{2}}\right] d x\right\} \\
= & \lim _{\varepsilon \downarrow 0}\left\{\left[e^{ \pm i \varepsilon\left(z-x^{\prime}\right)}-4 \frac{\alpha}{\varepsilon} \sin ^{2} \frac{\varepsilon}{2}\left(z-x^{\prime}\right) \pm i e^{ \pm 2 i \alpha z} \sin \varepsilon\left(z-x^{\prime}\right)\right] \psi_{0}\left(x^{\prime}\right)\right\}=\psi_{0}\left(x^{\prime}\right),
\end{aligned}
$$

where the upper (lower) signs correspond to the case $\operatorname{Im} z>0(\operatorname{Im} z<0)$. Hence, just the term

$$
\frac{2}{\pi \varepsilon \alpha} \sin ^{2} \frac{\varepsilon}{2}\left(x-x^{\prime}\right) \psi_{0}(x) \psi_{0}\left(x^{\prime}\right)
$$

in the resolutions of identity (3.8)-(3.10) gives an opportunity to reproduce $\psi_{0}(x)$ by these resolutions (cf. with the analogous results in Section 6.1 of [2], in Section 2 of [29] and in Section 2.4 of the present paper). It is interesting that the contribution of the term (3.13) in the resolutions of identity (3.8)-(3.10) is a singular discontinuous functional (see Remark 4.2 in [13]) which support consists of the only element - the infinity (cf. with the analogous comments in Section 2 of [29] and in Section 2.4 of the present paper).

There is another way to transform the resolution (3.7) as well. This way was used for obtaining of the resolutions of identity (26) in [29] and (2.28) in Section 2.3. The integral in the right-hand part of (3.7) is understood as follows,

$$
\int_{\mathcal{L}} \psi(x ; k) \psi\left(x^{\prime} ;-k\right) d k=\lim _{A \rightarrow+\infty}^{\prime} \int_{\mathcal{L}(A)} \psi(x ; k) \psi\left(x^{\prime} ;-k\right) d k,
$$

where $\mathcal{L}(A)$ is an integration path in complex $k$ plane, obtained from the segment $[-A, A]$ by its simultaneous deformation near the points $k=-\alpha$ and $k=\alpha$ upwards or downwards (the direction of this deformation is of no difference as well as in the case with $\mathcal{L}$ ) and the direction of $\mathcal{L}(A)$ is specified from $-A$ to $A$. Using

(1) the fact that the integral in the right-hand part of (3.14) is a standard integral (not a distribution);

(2) the fact that $(k \mp \alpha)^{2} \psi(x ; k) \psi\left(x^{\prime} ;-k\right)$ is a holomorphic function of $k$ in a neighborhood of $k= \pm \alpha($ see $(3.1))$;

(3) the Leibniz formula and the formulae (3.2);

(4) the notation $\mathcal{L}\left(k_{0} ; \varepsilon\right)$ with fixed $k_{0} \in \mathbb{R}$ and $\varepsilon>0$ for the path in complex $k$ plane defined by

$$
k=k_{0}+\varepsilon[\cos (\pi-\vartheta) \pm i \sin (\pi-\vartheta)], \quad 0 \leqslant \vartheta \leqslant \pi
$$

where the upper (lower) sign corresponds to the case of upper (lower) deformations in $\mathcal{L}$ and the direction of $\mathcal{L}\left(k_{0} ; \varepsilon\right)$ is specified from $\vartheta=0$ to $\vartheta=\pi$; 
we can transform (3.14) as follows,

$$
\begin{aligned}
\int_{\mathcal{L}} \psi(x ; & k) \psi\left(x^{\prime} ;-k\right) d k=\lim _{A \rightarrow+\infty}^{\prime} \lim _{\varepsilon \downarrow 0}\left\{\left(\int_{-A}^{-\alpha-\varepsilon}+\int_{-\alpha+\varepsilon}^{\alpha-\varepsilon}+\int_{\alpha+\varepsilon}^{A}\right) \psi(x ; k) \psi\left(x^{\prime} ;-k\right) d k\right. \\
& +\left.\sum_{j=0}^{1} \frac{1}{j !} \frac{\partial^{j}}{\partial k^{j}}\left[(k+\alpha)^{2} \psi(x ; k) \psi_{n}\left(x^{\prime} ;-k\right)\right]\right|_{k=-\alpha} \int_{\mathcal{L}(-\alpha ; \varepsilon)} \frac{d k}{(k+\alpha)^{2-j}} \\
& \left.+\left.\sum_{j=0}^{1} \frac{1}{j !} \frac{\partial^{j}}{\partial k^{j}}\left[(k-\alpha)^{2} \psi(x ; k) \psi_{n}\left(x^{\prime} ;-k\right)\right]\right|_{k=\alpha} \int_{\mathcal{L}(\alpha ; \varepsilon)} \frac{d k}{(k-\alpha)^{2-j}}\right\} \\
= & \lim _{A \rightarrow+\infty}^{\prime} \lim _{\varepsilon \downarrow 0}\left\{\left(\int_{-A}^{-\alpha-\varepsilon}+\int_{-\alpha+\varepsilon}^{\alpha-\varepsilon}+\int_{\alpha+\varepsilon}^{A}\right) \psi(x ; k) \psi\left(x^{\prime} ;-k\right) d k\right. \\
& +\frac{1}{4 \pi \alpha} \psi_{0}(x) \psi_{0}\left(x^{\prime}\right)\left[\int_{\mathcal{L}(-\alpha ; \varepsilon)} \frac{d k}{(k+\alpha)^{2}}+\int_{\mathcal{L}(\alpha ; \varepsilon)} \frac{d k}{(k-\alpha)^{2}}\right] \\
& \left.+\frac{1}{2 \pi}\left[\psi_{0}(x) \psi_{1}\left(x^{\prime}\right)+\psi_{1}(x) \psi_{0}\left(x^{\prime}\right)\right]\left[\int_{\mathcal{L}(\alpha ; \varepsilon)} \frac{d k}{k-\alpha}-\int_{\mathcal{L}(-\alpha ; \varepsilon)} \frac{d k}{k+\alpha}\right]\right\} \\
= & \lim _{A \rightarrow+\infty}^{\prime} \lim _{\varepsilon \downarrow 0}\left\{\left(\int_{-A}^{-\alpha-\varepsilon}+\int_{-\alpha+\varepsilon}^{\alpha-\varepsilon}+\int_{\alpha+\varepsilon}^{A}\right) \psi(x ; k) \psi\left(x^{\prime} ;-k\right) d k-\frac{1}{\pi \varepsilon \alpha} \psi_{0}(x) \psi_{0}\left(x^{\prime}\right)\right\} \\
= & \lim _{\varepsilon \downarrow 0}^{*}\left\{\left(\int_{-\infty}^{-\alpha-\varepsilon}+\int_{-\alpha+\varepsilon}^{\alpha-\varepsilon}+\int_{\alpha+\varepsilon}^{+\infty}\right) \psi(x ; k) \psi\left(x^{\prime} ;-k\right) d k-\frac{1}{\pi \varepsilon \alpha} \psi_{0}(x) \psi_{0}\left(x^{\prime}\right)\right\},(3.15)
\end{aligned}
$$

where the latter equality is considered as a definition for $\lim ^{*}$ and the limit $\lim _{\varepsilon \downarrow 0}$ is regarded as a pointwise one (not as a limit in a function space). The resolution of identity (3.15) is equivalent to (3.7), i.e. it is valid for all test functions for which (3.7) is valid (cf. with (3.11) and with the similar results in Section 2 of [29] and in Section 2.3 of the present paper).

Let us note that the associated function $\psi_{1}(x)$ does not appear in the derived resolutions of identity and is not expandable with the help of the resolution (3.11). Thereby, this associated function does not belong to the physical state space (rigged Hilbert space).

Let us notice also that the number (equal to 1) of linearly independent eigen- and (formal) associated functions of the Hamiltonian $h$ for the eigenvalue $E=\alpha^{2}$ included into the resolutions of identity (3.11) and (3.15) is less than the order (equal to 2) of the pole $E=\alpha^{2}$ of the Green function $G\left(x, x^{\prime} ; E\right)$.

\section{Conclusions: indexes of exceptional points and SUSY}

We remark that, in general, one can introduce, at least, three different number indexes of exceptional point $E=\lambda_{0}$ of a Hamiltonian $h$ :

(1) $n_{1}\left(\lambda_{0}\right)$ to be a maximal number of linearly independent normalizable eigenfunctions and associated functions of $h$ for the eigenvalue $E=\lambda_{0}$;

(2) $n_{2}\left(\lambda_{0}\right)$ to be a maximal number of linearly independent eigenfunctions and formal associated functions of $h$ for the eigenvalue $E=\lambda_{0}$ appeared in the resolution of identity constructed from biorthogonal set of eigenfunctions and associated functions of $h$;

(3) $n_{3}\left(\lambda_{0}\right)$ to be an order of the pole in the point $E=\lambda_{0}$ for the Green function for $h$ as a function of $E$ (in the case with the Hamiltonian $h=h_{n}$ (see Section 2), where the exceptional point $E=\lambda_{0}=0$ coincides with the branch point of the Green function; it is natural to assume (see Section 2.3) that $n_{3}(0)=n$ ). 
It can be proven (by methods of [7], see as well the example in Section 5.1 of [2]) that for an exceptional point outside of continuous spectrum all these indexes are identical,

$$
n_{1}\left(\lambda_{0}\right)=n_{2}\left(\lambda_{0}\right)=n_{3}\left(\lambda_{0}\right)
$$

and represent the algebraic multiplicity of the eigenvalue $\lambda_{0}$. In the cases where an exceptional point is situated on the border of continuous spectrum or inside of it these indexes may be different. For example, in the case of an exceptional point $E=0$ at the bottom of continuous spectrum of the Hamiltonian $h_{n}$ in Section 2,

$$
n_{1}(0)=\left[\frac{n+1}{2}\right], \quad n_{2}(0)=n, \quad n_{3}(0)=n \quad \Rightarrow \quad n_{1}(0) \leqslant n_{2}(0)=n_{3}(0)
$$

and in the case of an exceptional point $E=\alpha^{2}$ inside of continuous spectrum of the Hamiltonian $h$ in Section 3,

$$
n_{1}\left(\alpha^{2}\right)=1, \quad n_{2}\left(\alpha^{2}\right)=1, \quad n_{3}\left(\alpha^{2}\right)=2 \quad \Rightarrow \quad n_{1}\left(\alpha^{2}\right)=n_{2}\left(\alpha^{2}\right)<n_{3}\left(\alpha^{2}\right) .
$$

Thus, one can consider these indexes as different generalizations of the notion of algebraic multiplicity.

One can use SUSY technique $[14,15,16,17,18,19,20]$ in order to regulate the algebraic multiplicity (in any sense mentioned above) of an exceptional point in the spectrum of a SUSY partner Hamiltonian with respect to the order of this exceptional point in the spectrum of a given Hamiltonian (originally proposed in [21] and elaborated in details in [3, 4]):

(1) in order to increase the multiplicity of an exceptional point $\lambda_{0}$ in the spectrum of a SUSY partner Hamiltonian with respect to its order in the spectrum of a given Hamiltonian, one must take a formal eigenfunction (and a chain of formal associated functions) for the spectral value $\lambda_{0}$ of the latter Hamiltonian as transformation function(s) which tends (tend) to infinity for $x \rightarrow \pm \infty$;

(2) in order to decrease the multiplicity of an exceptional point $\lambda_{0}$ in the spectrum of a SUSY partner Hamiltonian with respect to its multiplicity in the spectrum of a given Hamiltonian, one must take a normalizable eigenfunction (and a chain of normalizable associated functions) of the latter Hamiltonian for eigenvalue $\lambda_{0}$ as transformation function(s).

These statements can be clarified by the following simple example. For the eigenvalue $E=\lambda_{0}=0$ of the Hamiltonian $h_{n}, n=1,2,3, \ldots$ in Section 2 there is a chain (2.1) of the eigenfunction $\psi_{n 0}(x)$ and associated functions $\psi_{n l}(x), l=1, \ldots,[(n-1) / 2]$. As well one can check that for the spectral value $E=0$ of the Hamiltonian $h_{n}, n=0,1,2, \ldots$ there is a chain of the formal eigenfunction $\varphi_{n 0}(x)$ and formal associated functions $\varphi_{n l}(x)$,

$$
\begin{aligned}
& \varphi_{n 0}(x)=(x-z)^{n+1}, \quad \varphi_{n l}(x)=\frac{(-1)^{l}(2 n+1) ! !}{(2 l) ! !(2 n+2 l+1) ! !}(x-z)^{n+2 l+1}, \\
& h_{n} \varphi_{n 0}=0, \quad h_{n} \varphi_{n l}=\varphi_{n, l-1}, \quad l=1,2,3, \ldots,
\end{aligned}
$$

which tend to infinity for $x \rightarrow \pm \infty$. Thus, it can be easily found that a) if to use $\varphi_{n l}(x)$, $l=0, \ldots, m$ as transformation functions for $h_{n}$, then the resulting Hamiltonian is $h_{n+m+1}$ with the exceptional point $E=0$ of larger algebraic multiplicity (except for the case with the indexes $n_{1}(0), m=0$ and odd $n$, where the indexes $n_{1}(0)$ for $h_{n}$ and $h_{n+m+1} \equiv h_{n+1}$ are equal), and b) if to use $\psi_{n l}(x), l=0, \ldots, m \leqslant[(n-1) / 2]$ as transformation functions for $h_{n}$, then the resulting Hamiltonian is $h_{n-m-1}$ with the exceptional point $E=0$ of smaller algebraic multiplicity (except for the case with indexes $n_{1}(0), m=0$ and even $n$, where the indexes $n_{1}(0)$ for $h_{n}$ and $h_{n-m-1} \equiv h_{n-1}$ are equal). 


\section{Acknowledgments}

This work was supported by Grant RFBR 09-01-00145-a and by the SPbSU project 11.0.64.2010. The work of A.A. was also supported by grants 2009SGR502, FPA2007-66665 and by the Consolider-Ingenio 2010 Program CPAN (CSD2007-00042).

\section{References}

[1] Berry M.V., Physics of non-Hermitian degeneracies, Czechoslovak J. Phys. 54 (2004), 1039-1047.

Heiss W.D., Exceptional points of non-Hermitian operators, J. Phys. A: Math. Gen. 37 (2004), 2455-2464, quant-ph/0304152.

Dembowski C., Dietz B., Gräf H.-D., Harney H.L., Heine A., Heiss W.D., Richter A., Encircling an exceptional point, Phys. Rev. E 69 (2004), 056216, 7 pages, nlin.CD/0402015.

Stehmann T., Heiss W.D., Scholtz F.G., Observation of exceptional points in electronic circuits, J. Phys. A: Math. Gen. 37 (2004), 7813-7819, quant-ph/0312182.

Mailybaev A.A., Kirillov O.N., Seyranian A.P., Geometric phase around exceptional points, Phys. Rev. A 72 (2005), 014104, 4 pages, quant-ph/0501040.

Müller M., Rotter I., Exceptional points in open quantum systems, J. Phys. A: Math. Theor. 41 (2008), 244018, 15 pages.

[2] Sokolov A.V., Andrianov A.A., Cannata F., Non-Hermitian quantum mechanics of non-diagonalizable Hamiltonians: puzzles with self-orthogonal states, J. Phys. A: Math. Gen. 39 (2006), 10207-10227, quant-ph/0602207.

[3] Andrianov A.A., Cannata F., Sokolov A.V., Non-linear supersymmetry for non-Hermitian, non-diagonalizable Hamiltonians. I. General properties, Nuclear Phys. B 773 (2007), 107-136, math-ph/0610024.

[4] Sokolov A.V., Non-linear supersymmetry for non-Hermitian, non-diagonalizable Hamiltonians. II. Rigorous results, Nuclear Phys. B $\mathbf{7 7 3}$ (2007), 137-171, math-ph/0610022.

[5] Klaiman S., Günther U., Moiseyev N., Visualization of branch points in $\mathcal{P} T$-symmetric waveguides, Phys. Rev. Lett. 101 (2008), 080402, 4 pages, arXiv:0802.2457.

Günther U., Samsonov B.F., The Naimark dilated PT-symmetric brachistochrone, Phys. Rev. Lett. 101 (2008), 230404, 4 pages, arXiv:0807.3643.

Günther U., Samsonov B.F., The $\mathcal{P} T$-symmetric brachistochrone problem, Lorentz boosts and non-unitary operator equivalence classes, Phys. Rev. A 78 (2008), 042115, 9 pages, arXiv:0709.0483.

[6] Pavlov B.S., On the spectral theory of non-selfadjoint differential operators, Dokl. Akad. Nauk SSSR 146 (1962), 1267-1270 (English transl.: Sov. Math. Dokl. 3 (1963), 1483-1487).

Pavlov B.S., On a non-self-adjoint Schrödinger operator, Probl. Math. Phys., Vol. 1, Spectral Theory and Wave Processes, Izdat. Leningrad Univ., Leningrad, 1966, 102-132 (in Russian).

[7] Naimark M.A., Linear differential operators, Frederick Ungar Publishing Co., New York, 1967.

[8] Heiss W.D., Phase transitions of finite Fermi systems and quantum chaos, Phys. Rep. 242 (1994), $443-451$. Heiss W.D., Müller M., Rotter I., Collectivity, phase transitions and exceptional points in open quantum systems, Phys. Rev. E 58 (1998), 2894-2901, quant-ph/9805038.

Narevicius E., Moiseyev N., Fingerprints of broad overlapping resonances in the $e+H_{2}$ cross section, Phys. Rev. Lett. 81 (1998), 2221-2224.

Narevicius E., Moiseyev N., Trapping of an electron due to molecular vibrations, Phys. Rev. Lett. 84 (2000), $1681-1684$.

[9] Schomerus H., Frahm K.M., Patra M., Beenakker C.W.J., Quantum limit of the laser linewidth in chaotic cavities and statistics of residues of scattering matrix poles, Phys. A 278 (2000), 469-496, chao-dyn/9911004.

[10] Hernández E., Jáuregui A., Mondragón A., Degeneracy of resonances in a double barrier potential, J. Phys. A: Math. Gen. 33 (2000), 4507-4523.

Dembowski C., Gräf H.-D., Harney H.L., Heine A., Heiss W.D., Rehfeld H., Richter A., Experimental observation of the topological structure of exceptional points, Phys. Rev. Lett. 86 (2001), 787-790.

[11] Bender C.M., Wu T.T., Analytic structure of energy levels in a field-theory model, Phys. Rev. Lett. 21 (1968), 406-409.

Bender C.M., Wu T.T., Anharmonic oscillator, Phys. Rev. 184 (1969), 1231-1260.

[12] Curtright T., Mezincescu L., Biorthogonal quantum systems, J. Math. Phys. 48 (2007), 092106, 35 pages, quant-ph/0507015.

Mostafazadeh A., Non-Hermitian Hamiltonians with a real spectrum and their physical applications, Pramana J. Phys. 73 (2009), 269-277, arXiv:0909.1654. 
[13] Sokolov A.V., Resolutions of identity for some non-Hermitian Hamiltonians. II. Proofs, SIGMA 7 (2011), 112, 16 pages, arXiv:1107.5916.

[14] Cooper F., Freedman B., Aspects of supersymmetric quantum mechanics, Ann. Physics 146 (1983), $262-288$.

[15] Fernández C. D.J., New hydrogen-like potentials, Lett. Math. Phys. 8 (1984), 337-343.

[16] Andrianov A.A., Borisov N.V., Ioffe M.V., Quantum systems with equivalent energy spectra, JETP Lett. 39 (1984), 93-97.

Andrianov A.A., Borisov N.V., Ioffe M.V., The factorization method and quantum systems with equivalent energy spectra, Phys. Lett. A 105 (1984), 19-22.

Andrianov A.A., Borisov N.V., Ioffe M.V., Factorization method and Darboux transformation for multidimensional Hamiltonians, Theoret. and Math. Phys. 61 (1985), 1078-1088.

[17] Sukumar C.V., Supersymmetry, factorisation of the Schrödinger equation and a Hamiltonian hierarchy, J. Phys. A: Math. Gen. 18 (1985), L57-L61.

Sukumar C.V., Supersymmetric quantum mechanics of one-dimensional systems, J. Phys. A: Math. Gen. 18 (1985), 2917-2936.

Sukumar C.V., Supersymmetric quantum mechanics and the inverse scattering method, J. Phys. A: Math. Gen. 18 (1985), 2937-2955.

[18] Bagrov V.G., Samsonov B.F., Darboux transformation of Schrödinger equation, Phys. Particles Nuclei 28 (1997), 374-397.

[19] Andrianov A.A., Cannata F., Nonlinear supersymmetry for spectral design in quantum mechanics, J. Phys. A: Math. Gen. 37 (2004), 10297-10321, hep-th/0407077.

[20] Aoyama H., Sato M., Tanaka T., $\mathcal{N}$-fold supersymmetry in quantum mechanics: general formalism, Nuclear Phys. B 619 (2001), 105-127, quant-ph/0106037.

[21] Samsonov B.F., Roy P., Is the $\mathcal{C} P T$ norm always positive?, J. Phys. A: Math. Gen. 38 (2005), L249-L255, quant-ph/0503040.

Samsonov B.F., SUSY transformations between diagonalizable and non-diagonalizable Hamiltonians, J. Phys. A: Math. Gen. 38 (2005), L397-L403, quant-ph/0503075.

[22] Fernández C. D.J., Fernández-García N., Higher-order supersymmetric quantum mechanics, AIP Conf. Proc. 744 (2005), 236-273, quant-ph/0502098.

[23] Gel'fand I.M., Vilenkin N.J., Generalized functions, Vol. 4, Some applications of harmonic analysis, Academic Press, New York, 1964.

[24] Bender C.M., Boettcher S., Real spectra in non-Hermitian Hamiltonians having $\mathcal{P} \mathcal{T}$ symmetry, Phys. Rev. Lett. 80 (1998), 5243-5246, physics/9712001.

Bender C.M., Boettcher S., Meisinger P., PT -symmetric quantum mechanics, J. Math. Phys. 40 (1999), 2201-2229, quant-ph/9809072.

Bender C.M., Brody D.C., Jones H.F., Complex extension of quantum mechanics, Phys. Rev. Lett. 89 (2002), 270401, 4 pages, Erratum, Phys. Rev. Lett. 92 (2004), 119902, 1 page, quant-ph/0208076.

Bender C.M., Chen J.H., Milton K.A., $\mathcal{P} \mathcal{T}$-symmetric versus Hermitian formulations of quantum mechanics, J. Phys. A: Math. Gen. 39 (2006), 1657-1668, hep-th/0511229.

Bender C.M., Making sense of non-Hermitian Hamiltonians, Rept. Progr. Phys. 70 (2007), 947-1018, hep-th/0703096.

[25] Schrödinger E., The factorization of the hypergeometric equation, Proc. Roy. Irish Acad. Sect. A 46 (1941), 53-54, physics/9910003.

[26] Infeld L., Hull T.E., The factorization method, Rev. Modern Phys. 23 (1951), 21-68.

[27] Andrianov A.A., Borisov N.V., Ioffe M.V., Eides M.I., Supersymmetric origin of equivalent quantum systems, Phys. Lett. A 109 (1985), 143-148.

Andrianov A.A., Borisov N.V., Ioffe M.V., Eides M.I., Supersymmetric mechanics: a new look at the equivalence of quantum systems, Theoret. and Math. Phys. 61 (1985), 965-972.

[28] Prudnikov A.P., Brychkov Yu.A., Marichev O.I., Integrals and series. Elementary functions, Nauka, Moscow, 1981 (in Russian).

[29] Andrianov A.A., Cannata F., Sokolov A.V., Spectral singularities for non-Hermitian one-dimensional Hamiltonians: puzzles with resolution of identity, J. Math. Phys. 51 (2010), 052104, 22 pages, arXiv:1002.0742. 